\title{
EL ESTADO DE SALUD DE LOS URUGUAYOS
} HEALTH STATUS OF URUGUAYAN PEOPLE

\author{
IANINA Rossi* \\ FERNANDA TELLECHEA \\ Fiorella TRAMONTIN \\ PATRICIA TRIUNFO
}

\begin{abstract}
Resumen
En el documento analizamos las vinculaciones entre estado de salud, medido mediante la autopercepción de la ocurrencia de enfermedades, y situación socioeconómica de los individuos. A través de la utilización de las Encuestas Continuas de Hogares de Uruguay de 1991 a 2000, estimamos modelos probit, encontrando que tienen una menor probabilidad de declarar un mal estado de salud los hombres, solteros, jóvenes, ocupados, quienes no viven solos y quienes tienen más de 5 años de educación. Asimismo, encontramos que la probabilidad de tener un mal estado de salud presenta una tendencia descendente en el período, pasando de 0,33 a 0,25.
\end{abstract}

\begin{abstract}
In this paper we analyze the links between health status (measured by selfreported illness occurrence) and individuals' socioeconomic situation. Using the annual households surveys of Uruguay for the period 1991 to 2000, we compute probit models and find that men, unmarried, young people, occupied, those who do not live alone and those with more than 5 years of education, have a smaller probability of reporting a bad health status. We also find that the probability of reporting a bad health status has a descendent trend in the period, falling from 0,33 in 1991 to 0,25 in 2000.
\end{abstract}

Key words: health capital, health status, human capital.

JEL Classification: I12, J24, D12.

* Departamento de Economía, Facultad de Ciencias Sociales, Universidad de la República, Uruguay. Web: http://www.decon.edu.uy.

E-Mails: ianina@decon.edu.uy; ftellechea@decon.edu.uy; ftramontin@bcu.gub.uy; patricia@decon.edu.uy 


\section{INTRODUCCIÓN}

La literatura sobre economía de la salud, en particular para países desarrollados, muestra que el estado de salud de los individuos está afectado no sólo por factores médicos sino también por variables socioeconómicas, tales como ingreso, educación, situación laboral, y factores ambientales, afectivos y emocionales.

En este sentido, en un estudio realizado para Suecia, utilizando datos del Level of Living Survey de 1991, Gerdtham y Johannesson (1997) aproximan el estado de salud mediante una variable categórica de autopercepción. Estimando modelos probit ordenados encuentran que la probabilidad de tener un buen estado de salud se reduce con la edad de los individuos, para quienes no están en pareja, tienen sobrepeso, viven en grandes ciudades, y para los hombres; a la vez que se incrementa con el ingreso y la educación.

Para Estados Unidos, Rhum (2001) utilizando microdatos de personas de 30 o más años entre 1972-1981, obtenidos de la National Health Interview Surveys, investiga la relación entre el estado de salud y la utilización de cuidados médicos con las condiciones macroeconómicas. Como aproximaciones al estado de salud el autor incluye el haber padecido determinadas enfermedades, el tener limitaciones de actividad, la autopercepción del estado general de salud, y la utilización de cuidados médicos, empleando diferentes metodologías de estimación de acuerdo a las características de la variable dependiente en cada caso: regresión lineal controlando por efectos fijos, estimación probit y estimación tobit. El principal resultado es que existe una relación negativa entre crecimiento económico y salud física, en especial para hombres y personas ocupadas. Por el contrario, la salud mental sería procíclica.

En este marco habría tres motivos por los cuales la salud podría empeorar cuando existe una buena condición económica: se reduce el tiempo dedicado a actividades no de mercado, aumenta el estrés relacionado con el trabajo, y el mayor ingreso puede llevar a que se realicen actividades recreativas más riesgosas para la salud.

Por su parte, Costa y Lahey (2003) utilizan datos longitudinales históricos de veteranos del Ejército de Estados Unidos que tenían entre 60 y 74 años de edad en el 1900 y de nacidos en Estados Unidos que tenían entre 60 y 79 años de edad entre 1960 y 1980, con el propósito de estudiar el rol del estrés ambiental en el ciclo de vida. Al analizar la notable disminución de la mortalidad de los adultos mayores en el mundo desarrollado, encuentran que los cambios en las condiciones físicas y sociales en las que los individuos viven son la principal causa de dicha disminución. En este sentido, estimando modelos probit y utilizando análisis de sobrevivencia, encuentran que aquellos nacidos en primavera, quienes vivían en grandes ciudades, y que pertenecían a un nivel socioeconómico bajo durante su niñez y juventud, vivieron menos años y tenían una probabilidad menor de llegar a la ancianidad.

A su vez, Fogel (2004), utilizando datos de la tasa de mortalidad infantil entre 1900 y 1950 para las seis ciudades más grandes de Estados Unidos, aplica modelos de sobrevivencia con tasas de riesgo en competencia, y encuentra que las condiciones de vida durante la infancia afectan el estado de morbilidad durante la adultez. De hecho, la cantidad de individuos que se ven afectados por una enfermedad crónica particular a edades específicas, y la edad a la que apa- 
recen las enfermedades crónicas, han disminuido durante el siglo XX. Según este autor, esto se relaciona con mejoras en el entorno y en el aumento del tamaño corporal, que derivan, principalmente, de condiciones socioeconómicas más favorables en el pasado reciente.

Respecto a la relación entre salud y educación, estudios que miden el estado de salud a través de morbilidad y mortalidad encuentran diferencias sustanciales según el nivel educativo. Esto se debería a que las personas con mayor nivel de instrucción suelen cuidarse más y ser más propensas a utilizar el sistema de salud, lo cual lleva a un aumento de la desigualdad en cuidados médicos y en los estados de salud individuales.

Sin embargo, Grignon (2005), utilizando información sobre la discapacidad corregida por la esperanza de vida ( "DALE"), los gastos en salud en dólares corregidos por PPP para 111 países (desarrollados y subdesarrollados) en el año 1997, y datos sobre la media de años de educación de la población de 15 y más años en 1995, a partir de los cuales estima distintas especificaciones $M C O$, encuentra que la educación tiene retornos decrecientes. De hecho, tiene un impacto nulo luego de traspasado un cierto umbral estimado en 8 años de educación curricular.

Otros estudios se centran en el efecto del ingreso y de la desigualdad del mismo sobre la salud. Por un lado, se plantea que el nivel de ingreso del país se encuentra positivamente relacionado con el estado de salud de los individuos, y, por otro lado, que la desigualdad en los ingresos de una sociedad se encuentra negativamente relacionada con el estado de salud de los individuos que la componen. Esto es de especial importancia si se toma en cuenta el hecho de que la demanda por cuidados médicos tiene una elasticidad ingreso relativamente alta.

No obstante, Deaton (2001), utilizando información de cortes por país sobre mortalidad de adultos en países ricos y mortalidad infantil en países pobres y ricos, encuentra resultados divergentes. Estimando modelos logit, concluye que la salud (aproximada con diferentes medidas de mortalidad como la tasa de mortalidad en el tiempo, los patrones geográficos de mortalidad y la mortalidad a nivel individual) estaría afectada por el ingreso absoluto y no por el relativo. Sin embargo, estas conclusiones pueden estar sesgadas por el hecho que el autor no cuenta con información confiable sobre la desigualdad del ingreso para los países en vías de desarrollo.

Existe escaso desarrollo del tema para países subdesarrollados. Para Uruguay, en particular, existe literatura económica que estudia la relación entre la salud de los individuos y las variables socioeconómicas para cohortes etarios específicos, no así análisis globales de la población ni evolución temporal.

En este sentido, centrándose en los adultos mayores montevideanos, Rossi y Triunfo (2004), utilizando la Encuesta Salud, Bienestar y Envejecimiento (SABE, MSP-OPS-OMS, 2001), estiman modelos probit ordenados y encuentran que los individuos con menor nivel educativo, los sedentarios, aquellos con deficiencias nutricionales, los que tienen solamente cobertura pública de salud, y las mujeres, tendrían una mayor probabilidad de tener un peor estado de salud, medido a través de las limitaciones funcionales, padecimiento de ciertas enfermedades crónicas y autorreporte de los individuos. Otra conclusión relevante es que las circunstancias vividas en las primeras etapas de la vida tienen un fuerte impacto en las condiciones de salud en la adultez. 
Jewell et al. (2006), utilizando la misma metodología, aproximaciones al estado de salud de los individuos y fuente de información, pero para los cinco países donde fue realizada (Argentina, Brasil, Chile, México y Uruguay), encuentran que la relación entre las medidas socioeconómicas y el autorreporte de salud es relativamente consistente entre estos cinco países. Los resultados indican que impacta positivamente en la salud, la buena nutrición, mayores niveles educativos, tener un estilo de vida activo y los que están satisfechos con su nivel de ingreso actual.

Para el cohorte etario extremo de los jóvenes de 14 a 19 años, Jewell et al. (2005) utilizan la Encuesta sobre Salud y Redes Sociales (dECON-UdelaR, 2004) y aproximan el estado de salud mediante una variable categórica de autopercepción. Estimando modelos probit ordenados encuentran que la probabilidad de tener un mejor estado de salud depende de la educación del joven, de su estado nutricional, de sus hábitos saludables (como el no fumar, realizar actividad física, etc.), de la ciudad donde vive (aumenta si vive en ciudades pequeñas), si cuenta con capital social, y de la privación relativa del hogar donde habita.

En este estudio, a través de la utilización de las Encuestas Continuas de Hogares (ECH) anuales del Instituto Nacional de Estadística (INE), relevadas durante el período 1991-2000, se analizará la relación entre el estado de salud de todos los uruguayos de 18 años y más -medido a través del autorreporte de los individuos- y variables socioeconómicas, mediante la estimación de modelos probit. Cabe destacar que la reformulación en el año 2001 de la ECH impide realizar dicho análisis hasta el momento actual.

\section{Un Modelo Teórico De Salud}

Siguiendo el modelo de inversión en salud de Grossman (1972a, 1972b, 2003), se asume que los individuos heredan un stock de salud inicial que se deprecia a lo largo del tiempo, y que puede ser incrementado por la inversión. Se trata de individuos racionales con información perfecta, por lo tanto, no existiría incertidumbre acerca de la ocurrencia ni de la duración de la enfermedad.

En este marco y según el autor, existen dos razones por las cuales los individuos demandan salud. Por un lado, forma parte de sus funciones de preferencias, es decir, la enfermedad genera desutilidad. Por otro lado, la salud determina el tiempo disponible para otras actividades, por lo tanto afecta tanto el ocio como el ingreso de los individuos.

Se supone que los individuos obtienen utilidad en cualquier período $t$ del ingreso y del stock de salud en el momento $t, H_{t}$. En cualquier período de tiempo $t, H_{t}$ es igual al stock de salud heredado del periodo anterior, $H_{t-1}$, más la inversión de salud realizada en dicho periodo, $I_{t-1}$, menos el monto de salud perdido debido a la depreciación. Por consiguiente, el stock de salud en cualquier periodo $t$ es el siguiente:

$$
\begin{gathered}
H_{t}=H_{t-1}+I_{t-1}-d_{t-1} H_{t-1} ; \mathrm{o} \\
H_{t}=\left(1-d_{t-1}\right) H_{t-1}+I_{t-1}
\end{gathered}
$$


Donde $d_{t-1}$ es la tasa de depreciación en el período $t-1$, la cual es un número entre 0 y 1 .

La maximización de la utilidad intertemporal sujeta a las restricciones presupuestales y a la ecuación (2) produce una ecuación para el stock óptimo de salud, por lo tanto, el monto óptimo de inversión en salud, en cualquier período de tiempo. Suponiendo que el individuo tiene el stock óptimo de salud en cada período, es posible resolver la ecuación (2) para el stock óptimo de salud en el período $T$. Sea $a_{t-1}$ igual a $1-d_{t-1}$ y $\theta$ el stock inicial de salud. Sustituyendo el óptimo $H_{t}$ para $t>T$ resulta en la siguiente condición, la cual describe el stock de salud del individuo en el período $T$ :

$$
H_{T}=\Pi_{i} a_{T-i} \theta+\Sigma_{j} I_{T-j} \Pi_{k} a_{T-k},
$$

donde $i=1, \ldots, T, j=1, \ldots, T-1, k=0, \ldots, j-1$, y $a_{T}=1$. Nótese que el stock de salud en el período $T$ es igual al valor depreciado del stock inicial de salud más la suma de los valores depreciados de todas las inversiones de salud hasta el período $T$.

Como se observa en la ecuación (3), $H_{T}$ es una función de las tasas de depreciación, de las inversiones en salud, y del stock inicial de salud del individuo, información que está contenida en la ecuación (4).

$$
H_{T}=F[d, I, \theta]
$$

En la forma general de la ecuación (4), $F$ es la forma de la función de $H_{T}$ (determinado por la relación entre depreciación en salud e inversión a lo largo del tiempo), $d$ es un vector de $T-1$ tasas de depreciación, e $I$ es un vector de $T-1$ inversiones en salud. Dado que el monto real de depreciación en salud e inversión es difícil de observar a lo largo del tiempo, muchos estudios estiman la ecuación (4) a través de aproximaciones para la depreciación y la inversión.

Asumiendo función lineal para $F$, tenemos que:

$$
H_{T}=\Omega \beta+\varepsilon,
$$

Donde $H_{T}$ es el capital actual de salud en el período $T$. Por otra parte, el vector $\Omega$ contiene las variables independientes (medidas de $d, I, y \theta$ ), el vector $\beta$ contiene los coeficientes a estimar, y $\varepsilon$ es el término de error.

En forma genérica, puede esperarse una relación negativa entre la variable que aproxima el buen estado de salud y las variables independientes que aumentan la depreciación del stock de salud, y una positiva con aquellas variables que incrementan la inversión en salud o su productividad. Dentro de las variables vinculadas a la depreciación, pueden citarse la edad, el consumo de sustancias nocivas para la salud, como las drogas, el tabaco y el alcohol, el sedentarismo, la dieta no balanceada, la práctica de actividades recreativas riesgosas, entre otras. Respecto de las variables que afectan la inversión en capital salud se encuentran factores socioeconómicos y culturales, como ser el sexo, el nivel educativo, el estado civil, el ingreso, etc. Influiría también aquí el capital social con el que cuenta el individuo, como las redes familiares y la calidad de los vínculos afectivos y estables que brindan seguridad y estabilidad emocional. 


\section{Metodología y Estrategias Empíricas}

El fenómeno que se pretende modelizar es discreto, tener un mal o buen estado de salud, por lo cual se estiman modelos de elección binaria. Siguiendo el modelo de Grossman (1972a, 1972b, 2003), el stock real de salud de los individuos en cualquier momento del tiempo constituye la variable no observada $\left(H^{*}\right)$, la cual se encuentra relacionada con el conjunto de variables independientes observadas $\left(x_{i}\right)$, que aproximan la depreciación y la inversión que realiza el individuo en capital salud: edad, sexo, estado civil, vivir solo, nivel educativo, condición de ocupación, cobertura de salud, ingreso.

$$
H^{*}{ }_{i}=\chi_{i} \beta+\varepsilon_{i}
$$

El vector de parámetros $\beta$ refleja el signo del impacto que las variables independientes tienen sobre la probabilidad de tener un buen o mal estado de salud. Un coeficiente positivo significa que la variable explicativa en cuestión aumenta la probabilidad de tener un mal estado de salud, y viceversa.

La relación entre la variable binaria observada $H$ y la latente $H^{*}$ está dada a través de las siguientes ecuaciones:

$$
H_{i}=\left\{\begin{array}{l}
1{\operatorname{si~} H^{*}}_{i}>0 \\
0 \operatorname{si~} H^{*}{ }_{i} \leq 0
\end{array}\right.
$$

Para un valor dado de $x$, la probabilidad de tener un mal estado de salud $(H=1)$ se puede escribir como:

$$
\operatorname{Pr}(H=1 / x)=F(x \beta) \text {, donde } \mathrm{F}(\cdot) \text { es la distribución acumulada }
$$

Mientras que la probabilidad de tener un buen estado de salud $(H=0)$ es:

$$
\operatorname{Pr}(H=0 / x)=1-F(x \beta)
$$

Si $F$ se distribuye normal, da lugar a los modelos denominados probit o probabilísticos que se utilizan en este trabajo:

$$
E(H / x)=0[1-F(x \beta)]+1[F(x \beta)]=F(x \beta)
$$

Tradicionalmente, el stock real de salud de los individuos se aproximaba mediante indicadores que recogían las pérdidas del mismo, como morbilidad y mortalidad, para cuya construcción se utilizaba la información proveniente de los servicios de salud y estadísticas vitales (INDEC (2003)). La ampliación de dicho concepto ha llevado a incorporar diferentes indicadores como ser la autopercepción de los individuos, enfermedades crónicas, limitaciones físicas, consultas médicas realizadas en un período, entre otros.

En el presente estudio se aproxima el estado de salud a través de la autopercepción de los individuos. Si bien se reconoce que esta medida puede estar sujeta a sesgos por endogeneidad y/o errores de medida, constituye un indicador confia- 
ble y muy frecuentemente utilizado para medir la salud de los individuos. ${ }^{1}$ Por otra parte, este tipo de medida discreta captura información importante y es un buen predictor de la mortalidad (Connelly et al. (1989); Idler y Kasl (1991)).

A partir de la información proveniente de las ECH del período 1991-2000, que incluyen una pregunta acerca de la autopercepción de la ocurrencia de enfermedades, se estima la probabilidad promedio de la población uruguaya mayor de 18 años de tener un mal estado de salud. Adicionalmente, se estudia la probabilidad de consultar, dado que el individuo se sintió enfermo.

Cabe destacar que la principal falencia de este tipo de estudios es la posible existencia de problemas de endogeneidad respecto a la educación, el ingreso, la condición de ocupación y la cobertura de salud, en el sentido de que no es posible descartar qué factores observables como inobservables determinen a dichas variables y a la salud a la vez. Desafortunadamente no es posible probar su existencia debido a las limitaciones de los datos. A dichos efectos debería estimarse una ecuación por cada variable endógena, siendo necesario contar con restricciones de exclusión que permitan identificar el sistema. ${ }^{2}$

En este estudio se optó por no utilizar el ingreso, aproximando el nivel socioeconómico de los individuos a través de su nivel educativo, condición de ocupación y cobertura de salud. Respecto a la educación, dado el cohorte etario que se analiza (individuos mayores de 18 años de edad), la totalidad de los individuos tiene la edad suficiente como para haber culminado la etapa formativa obligatoria ( 9 años de escolaridad en Uruguay), aunque aquellos entre 18 y 22 años no pueden encontrarse en el tramo superior. Sin embargo, se considera que la simultaneidad de los fenómenos está minimizada por existir apenas un $11 \%$ de la población que tiene entre 18 y 22 años, y por analizar la percepción del estado de salud de los últimos 12 meses. Por su parte, en lo que respecta a la condición de ocupación, existen instrumentos a los cuales pueden recurrir individuos con problemas de salud, como ser el seguro por enfermedad, en caso de dolencias de corta data, y los subsidios por incapacidad, en casos más serios.

Por otra parte, se tiene presente que en este tipo de estudios pueden existir sesgos de selección, en la medida que, al utilizar el autorreporte de los individuos, se analizan individuos que están vivos. De acuerdo a Heckman (1979) y Greene (1981), el uso de muestras no aleatoriamente seleccionadas para estimar relaciones de comportamiento deriva en errores de especificación que son resultado de la carencia de ciertos datos. Los datos de la muestra deberían ser tomados de una población mayor, individuos vivos y muertos. Según Heckman (1979), la carencia de datos lleva a estimadores inconsistentes, y establece que se pueden obtener estimadores consistentes si se realizan análisis probit para la muestra completa. Lo anterior implica estimar la probabilidad de que un individuo tenga un buen o mal estado de salud condicional a que está vivo.

1 Véase por ejemplo: Baker et al. (2001), Benítez-Silva et al. (2000), Bound (1991), Idler y Benyamini (1997), Mossey y Shapiro (1982), Rossi y Triunfo (2004), Salas (2002). Asimismo, existen trabajos recientes en Uruguay que se basan en otros indicadores y/o en fuentes de información diferentes a las que se utilizarán en el presente estudio, con los cuales se podría eventualmente realizar una comparación de resultados.

2 Se debe tener en cuenta que la literatura encuentra que muchas veces los sesgos de utilizar malas restricciones de exclusión son más graves que los que conlleva la endogeneidad. 
No obstante, existen estudios que controlando por las características socioeconómicas de los individuos que han fallecido, utilizando paneles completos e incompletos, encuentran que este sesgo de selección no es significativo (Salas (2002)).

Dado que no es posible aplicar la solución propuesta por Heckman (1979), puesto que en las ECH no se dispone de las características de los individuos que han fallecido, y dados los hallazgos de Salas (2002), se seguirá la solución planteada por Rossi y Triunfo (2004), que consiste en incluir en el análisis las variables que determinan la esperanza de vida de los individuos, como la edad y el sexo.

\section{Datos}

Se utilizan las ECH relevadas durante el período 1991-2000. Cabe destacar que en 1998 se introdujeron algunos cambios que podrían afectar la comparabilidad de los resultados obtenidos antes y después de dicho corte. Al respecto, Bucheli y Furtado (2001) citan la actualización de la muestra y la exclusión de las localidades con menos de 5.000 habitantes, de acuerdo a los datos del Censo de Población y Viviendas de 1996, y la modificación en el régimen de sustitución de viviendas.

El cambio en la muestra se caracterizó principalmente por la inclusión de la periferia de Montevideo y de las principales ciudades del interior del país. Por otra parte, se procedió a excluir las localidades entre 900 y 5.000 habitantes, y se modificó el criterio de selección de localidades. En este sentido, se estableció que aquellas con 15.000 o más habitantes debían a partir de entonces ser incluidas de forma obligatoria. Respecto al resto, es decir aquellas con 5.000 a 15.000 habitantes, se pasó a incluir una por cada departamento que posee por lo menos una localidad con estas características, con probabilidad conforme al número de habitantes. Por otra parte, se procedió a sustituir aquellas viviendas que no corresponden, es decir, que no se vinculan al menos a un hogar. Por último, se modificó de 3 a 6 las visitas fallidas necesarias para sustituir una vivienda válida por otra.

Con el objetivo de contar con muestras más comparables, se eliminarán de las ECH entre los años 1991 y 1997, a las localidades de menos de 5.000 habitantes. Igualmente, se tendrá especial precaución al analizar los cambios entre los años 1997 y 1998.

Las ECH del período 1991-2000 incluyen una pregunta acerca de la autopercepción del estado de salud de los individuos, respondida por un informante del hogar mayor de 18 años: “ ¿En los últimos 12 meses se sintió enfermo(a) y requirió asistencia médica de algún tipo? 1. No se sintió enfermo(a); 2. Se sintió enfermo(a), pero no requirió asistencia médica; 3 . Se sintió enfermo(a) y requirió asistencia médica en institución; 4. Se sintió enfermo(a) y requirió asistencia médica particular. De esta forma, la variable dependiente es construida a partir de esta pregunta y especificada como una variable binaria que toma el valor 1 cuando el individuo se ha sentido enfermo (mal estado de salud), y 0 en caso contrario (buen estado de salud), estimándose un modelo probit.

La distribución de la población según la atención médica requerida permite categorizar la variable dependiente en forma dicotómica (ver Cuadro 1). El 
porcentaje de la población de 18 y más años que no se sintió enferma ha aumentado en el decenio, pasando de un $66 \%$ en 1991 a un $73 \%$ en 2000. Cabe destacar que en el año 1999 el país entra en una recesión económica, cuyos efectos seguramente no son recogidos en la ECH del año 2000, dado que es dable esperar que se observe un cierto rezago entre el momento en que cae el nivel de actividad y el momento en que esto afecta la salud de los individuos.

Por otro lado, se observa que el porcentaje de personas que se sintieron enfermas pero no requirieron asistencia médica ha experimentado un decremento en el período de estudio, pasando de un $10 \%$ a un $4 \%$ de las personas que efectivamente se sintieron enfermas (ver Cuadro 2). No se dispone de datos acerca de las afecciones, como para saber si se trata de un cambio en las mismas, en la toma de conciencia de la población, o de disponibilidad de ingreso, etc. En este sentido, las personas podrían consultar más ante la ocurrencia de cualquier enfermedad cuando la economía se encuentra en expansión, por tener un mayor ingreso; mientras que al contar con menos dinero para tratar las enfermedades, los individuos podrían ser más tolerantes frente a las mismas.

\section{CUADRO 1}

AUTOPERCEPCIÓN DEL ESTADO DE SALUD. POBLACIÓN DE 18 AÑOS Y MÁS

(En porcentajes)

\begin{tabular}{|l|r|r|r|}
\cline { 2 - 4 } \multicolumn{1}{c|}{} & 1991 & 1995 & \multicolumn{1}{c|}{2000} \\
\hline No se sintió enfermo & 65.73 & 64.80 & 72.68 \\
\hline Se sintió enfermo pero no requirió asistencia médica & 3.54 & 1.52 & 1.07 \\
\hline $\begin{array}{l}\text { Se sintió enfermo y requirió asistencia médica en } \\
\text { institución }\end{array}$ & 30.05 & 33.40 & 25.90 \\
\hline $\begin{array}{l}\text { Se sintió enfermo y requirió asistencia médica } \\
\text { particular }\end{array}$ & 0.68 & 0.28 & 0.35 \\
\hline Total & 100.00 & 100.00 & 100.00 \\
\hline
\end{tabular}

Fuente: Elaboración propia en base a información de la ECH 1991-2000, INE.

CUADRO 2

PERSONAS QUE SE SINTIERON ENFERMAS EN EL PERÍODO. POBLACIÓN DE 18 AÑOS Y MÁS

(En porcentajes)

\begin{tabular}{|l|r|r|r|}
\cline { 2 - 4 } \multicolumn{1}{c|}{} & 1991 & 1995 & 2000 \\
\hline No requirió asistencia & 10.33 & 4.33 & 3.92 \\
\hline Sí requirió asistencia médica & 89.67 & 95.67 & 96.08 \\
\hline Total & 100.00 & 100.00 & 100.00 \\
\hline
\end{tabular}

Fuente: Elaboración propia en base a ECH 1991-2000, INE. 


\section{Variables independientes}

Las variables que se consideran inciden en el estado de salud de los individuos, se agrupan en características socioeconómicas y factores ambientales. De acuerdo al modelo de Grossman (1972a, 1972b, 2003), el primer grupo puede considerarse como el conjunto de variables que determinan la inversión del individuo en capital salud, dentro del cual se encuentran la edad, el sexo, el estado civil, la educación, el lugar de residencia y el capital social.

En relación a la edad se agrupa en cinco tramos etarios de manera de tener edades estadísticamente homogéneas dentro de cada grupo: de 18 a 25 años de edad (categoría omitida), 26 a 35, 36 a 45, 46 a 59, y 60 y más años. Se genera una variable binaria por tramo que adopta el valor 1 si el individuo pertenece a dicho grupo. Cabe destacar que la mayor proporción de individuos se encuentra en el tramo etario de 60 y más años, representando un $29 \%$ de la población mayor de 18 años en el año 2000.

El sexo se aproxima por una variable binaria que toma el valor 1 cuando el individuo es mujer, siendo en el año 2000, el 55\% de los casos de los mayores de 18 años de edad.

El estado civil se aproxima a través de cuatro variables binarias que toman el valor 1 si el individuo es soltero (categoría omitida), casado o en unión libre, divorciado o separado, o viudo. La mayor parte de la población mayor de 18 años se encuentra casada o viviendo en pareja, constituyendo un $57 \%$ en el año 2000 .

Respecto a la educación, se especifica mediante una variable continua de nivel educativo en años y la misma variable elevada al cuadrado. En el año 2000, el promedio de años de educación de la población mayor de 18 años es de 8.5 años.

Respecto a la condición de ocupación, se define una serie de variables binarias que toman el valor 1 si el individuo cumple con la condición especificada: ocupado (categoría omitida), desocupado o inactivo. Dichos grupos corresponden al 55\%, 8\% y 37\% de la población mayor de 18 años en el año 2000, respectivamente.

En relación al tipo de cobertura, dado que el mismo es diferente en Montevideo que en el resto del país, se opta por definir variables que tienen en cuenta ambas características en forma conjunta. En este sentido, se definen cinco variables binarias: cobertura pública en Montevideo (16\% de la población mayor de 18 años en el año 2000), cobertura pública en el interior (24\%), cobertura privada en Montevideo (37\%), cobertura privada en el interior (18\%), y sin cobertura (5\%). Se omite del análisis la variable correspondiente a este último grupo. Debido a la forma en que son definidas estas variables, no se incluyen variables binarias por zona geográfica.

En cuanto al capital social, por no disponer de otra información se lo aproxima a través de la cantidad de personas que viven en el hogar, especificando una variable binaria que toma el valor $1 \mathrm{si}$ el individuo vive solo en el hogar, siendo $8 \%$ en el año 2000.

Como variables que aproximan la depreciación del stock de capital salud, se considera únicamente la edad por carecer de datos sobre los hábitos y conductas del individuo, como ser el sedentarismo, consumo de sustancias nocivas, dieta no balanceada, etc. 
Por último, se incorporan variables binarias por año, con el objetivo de evaluar la evolución en el tiempo. Los Cuadros 3 y 4 presentan las definiciones de las variables utilizadas y sus estadísticas descriptivas.

\section{CUADRO 3}

DEFINICIÓN DE LAS VARIABLES UTILIZADAS.

ECH 1991-2000

\begin{tabular}{|c|c|}
\hline Variables & Definición \\
\hline Mal estado de salud & $=1 \mathrm{si}$ el individuo se sintió enfermo \\
\hline Consulta & $\begin{array}{l}=1 \text { si el individuo se sintió enfermo y requirió } \\
\text { asistencia médica }\end{array}$ \\
\hline Edad 18-25 (omitida) & $=1$ si tiene entre 18 y 25 años \\
\hline Edad 26-35 & $=1$ si tiene entre 26 y 35 años \\
\hline Edad 36-45 & $=1$ si tiene entre 36 y 45 años \\
\hline Edad 46-59 & $=1$ si tiene entre 46 y 59 años \\
\hline Edad 60 y más & $=1$ si tiene 60 o más años \\
\hline No cobertura (omitida) & $=1$ si no tiene cobertura de salud \\
\hline Pública Montevideo & $\begin{aligned} &= 1 \text { si tiene cobertura de salud pública y vive en } \\
& \text { Montevideo }\end{aligned}$ \\
\hline Pública Interior & $\begin{array}{l}=1 \text { si tiene cobertura de salud pública y vive en el } \\
\text { interior }\end{array}$ \\
\hline Privada Montevideo & $\begin{array}{l}=1 \text { si tiene cobertura de salud privada y vive en } \\
\text { Montevideo }\end{array}$ \\
\hline Privada Interior & $\begin{array}{l}=1 \text { si tiene cobertura de salud privada y vive en el } \\
\text { interior }\end{array}$ \\
\hline Ocupado (omitida) & $=1$ si es ocupado \\
\hline Desocupado & $=1$ si es desempleado \\
\hline Inactivo & $\begin{aligned}= & 1 \text { si es jubilado, pensionista, estudiante, ama de casa, } \\
& \text { desalentado }\end{aligned}$ \\
\hline Mujer & $=1$ si es mujer \\
\hline Soltero (omitida) & $=1 \mathrm{si}$ es soltero \\
\hline Casado & $=1$ si es casado o en unión libre \\
\hline Divorciado & $=1$ si es divorciado o separado \\
\hline Viudo & $=1 \mathrm{si}$ es viudo \\
\hline Vive Solo & $=1$ si la cantidad de personas en el hogar es 1 \\
\hline Educación & $=$ años de educación \\
\hline Educación ${ }^{2}$ & $=($ años de educación $) *($ años de educación $)$ \\
\hline
\end{tabular}

Fuente: Elaboración propia en base a la ECH 1991-2000, INE. 


\section{CUADRO 4}

ESTADÍSTICAS DESCRIPTIVAS DE LAS VARIABLES UTILIZADAS,

PRESENTÁNDOSE LAS MEDIAS EN LAS CELDAS SUPERIORES Y LOS DESVÍOS ESTÁNDAR EN LAS CELDAS INFERIORES

(Población de 18 y más años de edad)

\begin{tabular}{|c|c|c|c|c|c|c|c|c|c|c|}
\hline Año & 1991 & 1992 & 1993 & 1994 & 1995 & 1996 & 1997 & 1998 & 1999 & 2000 \\
\hline \multirow{2}{*}{ Mal estado } & 0.3427 & 0.3438 & 0.3364 & 0.3340 & 0.3521 & 0.3196 & 0.3167 & 0.2897 & 0.2765 & 0.2732 \\
\hline & 0.4746 & 0.4750 & 0.4725 & 0.4717 & 0.4776 & 0.4663 & 0.4652 & 0.4536 & 0.4473 & 0.4456 \\
\hline \multirow{2}{*}{ Consulta } & 0.8967 & 0.8755 & 0.9208 & 0.9311 & 0.9567 & 0.9484 & 0.9066 & 0.9032 & 0.9316 & 0.9608 \\
\hline & 0.3044 & 0.3302 & 0.2701 & 0.2533 & 0.2035 & 0.2213 & 0.2909 & 0.2956 & 0.2525 & 0.1941 \\
\hline \multirow{2}{*}{ Edad 18-25 } & 0.1566 & 0.1576 & 0.1610 & 0.1661 & 0.16987 & 0.1730 & 0.1719 & 0.1700 & 0.1659 & 0.1727 \\
\hline & 0.3634 & 0.3643 & 0.3675 & 0.3722 & 0.3753 & 0.3782 & 0.3773 & 0.3756 & 0.3720 & 0.3780 \\
\hline \multirow{2}{*}{ Edad 26-35 } & 0.1774 & 0.1747 & 0.1649 & 0.1689 & 0.1671 & 0.1628 & 0.1628 & 0.1772 & 0.1686 & 0.1654 \\
\hline & 0.3820 & 0.3797 & 0.3711 & 0.3747 & 0.3731 & 0.3692 & 0.3692 & 0.3818 & 0.3744 & 0.3715 \\
\hline \multirow{2}{*}{ Edad 36-45 } & 0.1667 & 0.1675 & 0.1672 & 0.1728 & 0.1729 & 0.1702 & 0.1694 & 0.1800 & 0.1757 & 0.1749 \\
\hline & 0.3727 & 0.3734 & 0.3731 & 0.3781 & 0.3782 & 0.3758 & 0.3751 & 0.3842 & 0.3806 & 0.3799 \\
\hline \multirow{2}{*}{ Edad 46-59 } & 0.2104 & 0.2131 & 0.2043 & 0.2083 & 0.2076 & 0.2107 & 0.2057 & 0.2024 & 0.2020 & 0.2004 \\
\hline & 0.4076 & 0.4095 & 0.4032 & 0.4061 & 0.4056 & 0.4078 & 0.4042 & 0.4018 & 0.4015 & 0.4003 \\
\hline \multirow{2}{*}{ Edad $60+$} & 0.2890 & 0.2872 & 0.3026 & 0.2838 & 0.2827 & 0.2833 & 0.2902 & 0.2705 & 0.2879 & 0.2867 \\
\hline & 0.4533 & 0.4525 & 0.4594 & 0.4509 & 0.4503 & 0.4506 & 0.4539 & 0.4442 & 0.4528 & 0.4522 \\
\hline \multirow{2}{*}{ Sin cobertura } & 0.0609 & 0.0636 & 0.0590 & 0.0571 & 0.0617 & 0.0549 & 0.0562 & 0.0490 & 0.0472 & 0.0464 \\
\hline & 0.2391 & 0.2440 & 0.2356 & 0.2322 & 0.2407 & 0.2278 & 0.2303 & 0.2159 & 0.2121 & 0.2103 \\
\hline \multirow{2}{*}{$\begin{array}{l}\text { Pública } \\
\text { Montevideo }\end{array}$} & 0.1046 & 0.0946 & 0.1038 & 0.1112 & 0.1146 & 0.1256 & 0.1331 & 0.1374 & 0.1448 & 0.1556 \\
\hline & 0.3061 & 0.2927 & 0.3050 & 0.3144 & 0.3185 & 0.3314 & 0.3397 & 0.3443 & 0.3519 & 0.3625 \\
\hline \multirow{2}{*}{$\begin{array}{l}\text { Pública } \\
\text { Interior }\end{array}$} & 0.1868 & 0.1941 & 0.2060 & 0.2148 & 0.2269 & 0.2311 & 0.2377 & 0.2448 & 0.2328 & 0.2435 \\
\hline & 0.3897 & 0.3955 & 0.4045 & 0.4107 & 0.4188 & 0.4216 & 0.4257 & 0.4300 & 0.4226 & 0.4292 \\
\hline \multirow{2}{*}{$\begin{array}{l}\text { Privada } \\
\text { Montevideo }\end{array}$} & 0.4058 & 0.4135 & 0.4137 & 0.3991 & 0.3741 & 0.3760 & 0.3630 & 0.3366 & 0.3842 & 0.3730 \\
\hline & 0.4910 & 0.4925 & 0.4925 & 0.4897 & 0.4839 & 0.4844 & 0.4809 & 0.4726 & 0.4864 & 0.4836 \\
\hline \multirow{2}{*}{$\begin{array}{l}\text { Privada } \\
\text { Interior }\end{array}$} & 0.2419 & 0.2342 & 0.2175 & 0.2177 & 0.2227 & 0.2123 & 0.2101 & 0.2321 & 0.1910 & 0.1815 \\
\hline & 0.4283 & 0.4235 & 0.4125 & 0.4127 & 0.4161 & 0.4090 & 0.4074 & 0.4222 & 0.3931 & 0.3854 \\
\hline \multirow{2}{*}{ Mujer } & 0.5432 & 0.5495 & 0.5515 & 0.5478 & 0.5481 & 0.5475 & 0.5452 & 0.5433 & 0.5460 & 0.5452 \\
\hline & 0.4981 & 0.4976 & 0.4974 & 0.4977 & 0.4977 & 0.4977 & 0.4980 & 0.4981 & 0.4979 & 0.4980 \\
\hline \multirow{2}{*}{ Soltero } & 0.2294 & 0.2167 & 0.2381 & 0.2282 & 0.2317 & 0.2365 & 0.2373 & 0.2374 & 0.2487 & 0.2518 \\
\hline & 0.4204 & 0.4120 & 0.4259 & 0.4197 & 0.4219 & 0.4250 & 0.4254 & 0.4255 & 0.4323 & 0.4341 \\
\hline \multirow{2}{*}{ Casado } & 0.6154 & 0.6209 & 0.5975 & 0.6083 & 0.5969 & 0.5939 & 0.5874 & 0.5955 & 0.5794 & 0.5736 \\
\hline & 0.4865 & 0.4852 & 0.4904 & 0.4881 & 0.4905 & 0.4911 & 0.4923 & 0.4908 & 0.4937 & 0.4946 \\
\hline \multirow{2}{*}{ Divorciado } & 0.0602 & 0.0619 & 0.0598 & 0.0609 & 0.0686 & 0.0695 & 0.0724 & 0.0730 & 0.0752 & 0.0781 \\
\hline & 0.2378 & 0.2410 & 0.2370 & 0.2392 & 0.2527 & 0.2543 & 0.2592 & 0.2601 & 0.2637 & 0.2684 \\
\hline \multirow{2}{*}{ Viudo } & 0.0951 & 0.1005 & 0.1046 & 0.1026 & 0.1029 & 0.1001 & 0.1029 & 0.0941 & 0.0967 & 0.0964 \\
\hline & 0.2933 & 0.3007 & 0.3061 & 0.3034 & 0.3038 & 0.3002 & 0.3038 & 0.2919 & 0.2956 & 0.2952 \\
\hline \multirow[t]{2}{*}{ Vive solo } & 0.0582 & 0.0752 & 0.0641 & 0.0625 & 0.0608 & 0.0653 & 0.0668 & 0.0675 & 0.0745 & 0.0761 \\
\hline & 0.2341 & 0.2637 & 0.2450 & 0.2420 & 0.2390 & 0.2470 & 0.2497 & 0.2509 & 0.2625 & 0.2651 \\
\hline \multirow[t]{2}{*}{ Ocupado } & 0.5614 & 0.5589 & 0.5564 & 0.5622 & 0.5647 & 0.5485 & 0.5461 & 0.5763 & 0.5659 & 0.5552 \\
\hline & 0.4962 & 0.4965 & 0.4968 & 0.4961 & 0.4958 & 0.4976 & 0.4979 & 0.4942 & 0.4956 & 0.4970 \\
\hline \multirow{2}{*}{ Desocupado } & 0.0470 & 0.0471 & 0.0431 & 0.0479 & 0.0561 & 0.0659 & 0.0643 & 0.0596 & 0.0653 & 0.0788 \\
\hline & 0.2117 & 0.2119 & 0.2030 & 0.2136 & 0.2301 & 0.2482 & 0.2453 & 0.2367 & 0.2471 & 0.2694 \\
\hline Inactivo & 0.3916 & 0.3940 & 0.4006 & 0.3899 & 0.3792 & 0.3855 & 0.3896 & 0.3641 & 0.3688 & 0.3660 \\
\hline mactivo & 0.4881 & 0.4886 & 0.4900 & 0.4877 & 0.4852 & 0.4867 & 0.4877 & 0.4812 & 0.4825 & 0.4817 \\
\hline Educación & 7.5852 & 7.8018 & 7.7443 & 7.9234 & 8.0525 & 8.1636 & 8.1511 & 8.2675 & 8.4097 & 8.5198 \\
\hline Eaucacion & 4.0906 & 4.0208 & 4.1105 & 3.9970 & 4.0272 & 4.0259 & 3.9800 & 4.0279 & 4.1816 & 4.1470 \\
\hline & 74.2686 & 77.0349 & 76.8707 & 78.7556 & 81.0603 & 82.8524 & 82.2808 & 84.5753 & 88.2087 & 89.7845 \\
\hline Educacion² & 73.9869 & 74.3359 & 74.6250 & 74.4557 & 75.7729 & 76.4020 & 74.9666 & 76.8629 & 79.7524 & 80.2479 \\
\hline
\end{tabular}

Fuente: Elaboración propia en base a ECH 1991-2000, INE.

Nota: En el caso de variables binarias, la media equivale a la frecuencia. Para Educación, el mínimo es 0 y el máximo es 21 . 


\section{Resultados}

Se estima la ecuación (5) a través de un modelo probit. En el Cuadro 5 se presentan las estimaciones con los errores estándares robustos. Dado que el modelo no es lineal sólo es posible analizar directamente el signo de los coeficientes pero no su magnitud, estimándose para ello los efectos marginales (ver Cuadros 6 y 7 ).

Cabe resaltar que la mayor carencia de este tipo de estudio es la imposibilidad de controlar por endogeneidad, debido a la inexistencia de restricciones de exclusión adecuadas. Por tal motivo, no es posible conocer el sentido de la causalidad entre las variables del modelo, sino correlaciones entre las mismas.

Con el objetivo de verificar si es posible trabajar con el pool data, se realizaron pruebas de Chow. Se rechaza la hipótesis de que los coeficientes sean iguales por año $($ Chi2 $(8)=1265.96$ con $\operatorname{Prob}>\operatorname{chi} 2=0.0000, \mathrm{n}=426097)$, por lo que se realizan las estimaciones año por año, teniendo especial precaución al analizar los valores predichos antes y después de 1998, momento en que cambió la forma de relevar la información. ${ }^{3}$

En primer lugar, se analiza la probabilidad promedio de tener un mal estado de salud por año (ver Gráfico 1). La misma presenta una tendencia decreciente en el período, a excepción del año 1995 , situándose entre 0.33 y 0.25 . Teniendo en cuenta que el estado de salud es una variable relativamente estable en el tiempo, y que se está analizando la variación en un año, la magnitud de esta caída parece considerable. Sin embargo, no es posible atribuir esta variación a un cambio efectivo en la probabilidad de tener un mal estado de salud, por los motivos mencionados anteriormente.

Respecto de las variables explicativas, para todos los años analizados, se obtiene que la edad sería un fuerte indicador de depreciación del stock de salud de los individuos, confirmando la hipótesis teórica y en concordancia con la evidencia relevada tanto en países desarrollados como en vías de desarrollo. En este sentido, se observa que la probabilidad de tener un mal estado de salud aumenta notoriamente con el pasaje de un tramo etario a otro mayor.

El análisis se realiza respecto del tramo etario omitido, de 18 a 25 años de edad. El tener entre 26 y 35 años tiene un efecto marginal moderado, oscilando entre 1 y 6 puntos porcentuales en el período analizado. No obstante, dicha variable no resulta significativa para los años 1997, 1999 y 2000. Se trata de un resultado esperable, puesto que las complicaciones en la salud suelen en general presentarse luego de pasados los 35 años de edad. Por otra parte, el tener 60 o más años tiene una correlación fuertemente positiva con el mal estado de salud, teniendo un efecto marginal en torno a los 25 puntos porcentuales durante el período. De hecho, al analizar tramos etarios extremos se encuentra que la probabilidad de tener un mal estado de salud en los adultos mayores más que duplica la de los jóvenes entre 18 y 25 años.

En relación a la condición de ocupación, se constata que en todos los casos que las variables que representan el estar desocupado y el ser inactivo son significativas y presentan el signo esperado, es decir, que existe una asociación positiva entre éstas y la probabilidad de un mal estado de salud.

3 Este hallazgo imposibilitó el uso de restricciones de exclusión relacionadas con cambios institucionales. 


\section{CUADRO 5}

RESULTADOS DE LAS ESTIMACIONES PROBIT. VARIABLE DEPENDIENTE: ESTADO DE SALUD ( $1=$ MAL ESTADO, $0=$ BUEN ESTADO $)$

(Población de 18 y más años de edad)

\begin{tabular}{|c|c|c|c|c|c|c|c|c|c|c|}
\hline $\begin{array}{l}\text { Año } \\
\mathrm{N}^{\circ} \text { obs }\end{array}$ & $\begin{array}{c}1991 \\
41770\end{array}$ & $\begin{array}{c}1992 \\
41759\end{array}$ & $\begin{array}{c}1993 \\
42435\end{array}$ & $\begin{array}{c}1994 \\
42746\end{array}$ & $\begin{array}{c}1995 \\
44214\end{array}$ & $\begin{array}{c}1996 \\
44306\end{array}$ & $\begin{array}{c}1997 \\
44067\end{array}$ & $\begin{array}{c}1998 \\
40382\end{array}$ & $\begin{array}{c}1999 \\
41624\end{array}$ & $\begin{array}{c}2000 \\
42061\end{array}$ \\
\hline \multirow[t]{2}{*}{$\begin{array}{l}\text { Edad } \\
26-35\end{array}$} & $\begin{array}{c}0.1275 \\
(* *)\end{array}$ & $\begin{array}{c}0.0673 \\
(* *)\end{array}$ & $\begin{array}{c}0.0740 \\
(* *)\end{array}$ & $\begin{array}{c}0.0821 \\
(* * *)\end{array}$ & $\begin{array}{c}0.1631 \\
(* * *)\end{array}$ & $\begin{array}{c}0.0469 \\
(*)\end{array}$ & 0.0365 & $\begin{array}{c}0.0478 \\
(*)\end{array}$ & 0.0269 & 0.0444 \\
\hline & 0.0264 & 0.0265 & 0.0271 & 0.0268 & 0.0254 & 0.0260 & 0.0259 & 0.0269 & 0.0271 & 0.0273 \\
\hline \multirow[t]{2}{*}{$\begin{array}{l}\text { Edad } \\
36-45\end{array}$} & $\begin{array}{c}0.2046 \\
(* *)\end{array}$ & $\begin{array}{c}0.1120 \\
(* * *)\end{array}$ & $\begin{array}{c}0.2003 \\
(* *)\end{array}$ & $\begin{array}{c}0.1864 \\
(* * *)\end{array}$ & $\begin{array}{c}0.2248 \\
(* *)\end{array}$ & $\begin{array}{c}0.1016 \\
(* * *)\end{array}$ & $\begin{array}{c}0.1274 \\
(* *)\end{array}$ & 0.0339 & $\begin{array}{c}0.1253 \\
(* * *)\end{array}$ & $\begin{array}{c}0.0940 \\
(* * *)\end{array}$ \\
\hline & 0.0277 & 0.0280 & 0.0281 & 0.0280 & 0.0267 & 0.0273 & 0.0270 & 0.0284 & 0.0283 & 0.0285 \\
\hline \multirow[t]{2}{*}{$\begin{array}{l}\text { Edad } \\
46-59\end{array}$} & $\begin{array}{c}0.3820 \\
(* *)\end{array}$ & $\begin{array}{c}0.3039 \\
(* *)\end{array}$ & $\begin{array}{c}0.4164 \\
(* *)\end{array}$ & $\begin{array}{c}0.4015 \\
(* * *)\end{array}$ & $\begin{array}{c}0.3836 \\
(* *)\end{array}$ & $\begin{array}{c}0.3165 \\
(* * *)\end{array}$ & $\begin{array}{c}0.2892 \\
(* *)\end{array}$ & $\begin{array}{c}0.2558 \\
(* *)\end{array}$ & $\begin{array}{c}0.2676 \\
(* *)\end{array}$ & $\begin{array}{c}0.2868 \\
(* * *)\end{array}$ \\
\hline & 0.0271 & 0.0271 & 0.0273 & 0.0271 & 0.0261 & 0.0263 & 0.0262 & 0.0277 & 0.0276 & 0.0278 \\
\hline \multirow{2}{*}{$\begin{array}{l}\text { Edad } \\
60+\end{array}$} & $\begin{array}{c}0.6818 \\
(* *)\end{array}$ & $\begin{array}{c}0.5959 \\
(* * *)\end{array}$ & 0.7918 & $\begin{array}{c}0.7339 \\
(* * *)\end{array}$ & $\begin{array}{c}0.7604 \\
* * *)\end{array}$ & $\begin{array}{l}0.6074 \\
* * *)\end{array}$ & $\begin{array}{l}0.6071 \\
(* * *)\end{array}$ & 0.5436 & $\begin{array}{c}0.6427 \\
(* * *)\end{array}$ & $\begin{array}{c}0.6358 \\
* * *)\end{array}$ \\
\hline & 0.0286 & 0.0285 & 0.0288 & 0.0288 & 0.0279 & 0.0280 & 0.0279 & 0.0297 & 0.0294 & 0.0293 \\
\hline \multirow{2}{*}{$\begin{array}{l}\text { Pública } \\
\text { Mvd }\end{array}$} & 0.3919 & 0.3594 & 0.4757 & 0.6101 & 0.6374 & 0.6197 & 0.4596 & 0.2676 & 0.2133 & 0.2854 \\
\hline & $\begin{array}{c}(* * *) \\
0.0363\end{array}$ & $\begin{array}{c}(* * *) \\
0.0364\end{array}$ & $\begin{array}{c}(* * *) \\
0.0378\end{array}$ & $\begin{array}{c}(* * *) \\
0.0376\end{array}$ & $\begin{array}{c}(* * *) \\
0.0352\end{array}$ & $\begin{array}{c}(* * *) \\
0.0380\end{array}$ & $\begin{array}{c}(* * *) \\
0.0367\end{array}$ & $\begin{array}{c}(* * *) \\
0.0403\end{array}$ & $\begin{array}{c}(* * *) \\
0.0412\end{array}$ & $\begin{array}{l}(* * *) \\
0.0431\end{array}$ \\
\hline \multirow{2}{*}{$\begin{array}{l}\text { Pública } \\
\text { Interior }\end{array}$} & 0.3459 & 0.2890 & 0.3549 & 0.2856 & 0.3200 & 0.3828 & 0.3321 & 0.3965 & 0.4452 & 0.5332 \\
\hline & 0.0338 & 0.0333 & 0.0353 & 0.0356 & 0.0331 & 0.0364 & 0.0351 & 0.0383 & 0.0396 & 0.0415 \\
\hline \multirow{2}{*}{$\begin{array}{l}\text { Privada } \\
\text { Mvd }\end{array}$} & $\begin{array}{c}0.4645 \\
(* * *)\end{array}$ & $\begin{array}{c}0.4958 \\
(* *)\end{array}$ & $\begin{array}{c}0.5379 \\
(* * *)\end{array}$ & $\begin{array}{c}0.6807 \\
(* *)\end{array}$ & $\begin{array}{c}0.6677 \\
(* * *)\end{array}$ & $\begin{array}{c}0.7049 \\
(* *)\end{array}$ & 0.5312 & $\begin{array}{c}0.3374 \\
(* * *)\end{array}$ & $\begin{array}{c}0.3319 \\
(* *)\end{array}$ & 0.4129 \\
\hline & 0.0321 & 0.0318 & 0.0339 & 0.0343 & 0.0321 & 0.0356 & 0.0343 & 0.0381 & 0.0389 & 0.0412 \\
\hline \multirow{2}{*}{$\begin{array}{l}\text { Privada } \\
\text { Interior }\end{array}$} & 0.5133 & 0.4114 & 0.4776 & 0.3568 & 0.4174 & 0.5306 & 0.5266 & 0.5161 & 0.5900 & 0.7545 \\
\hline & 0.0330 & 0.0329 & 0.0351 & 0.0356 & 0.0332 & 0.0366 & 0.0353 & 0.0386 & 0.0400 & 0.0422 \\
\hline \multirow[t]{2}{*}{$\begin{array}{l}\text { Desocu- } \\
\text { pado }\end{array}$} & $\begin{array}{c}0.0869 \\
(* *)\end{array}$ & $\begin{array}{c}0.1147 \\
(* * *)\end{array}$ & $\begin{array}{c}0.1397 \\
(* *)\end{array}$ & $\begin{array}{c}0.1317 \\
(* * *)\end{array}$ & $\begin{array}{c}0.1232 \\
(* * *)\end{array}$ & $\begin{array}{c}0.1168 \\
(* * *)\end{array}$ & $\begin{array}{c}0.1024 \\
(* *)\end{array}$ & $\begin{array}{c}0.1568 \\
(* *)\end{array}$ & $\begin{array}{c}0.1227 \\
(* * *)\end{array}$ & $\begin{array}{c}0.0788 \\
(* * *)\end{array}$ \\
\hline & 0.0341 & 0.0338 & 0.0357 & 0.0336 & 0.0301 & 0.0284 & 0.0289 & 0.0311 & 0.0299 & 0.0285 \\
\hline \multirow{2}{*}{ Inactivo } & 0.1931 & 0.2179 & 0.2467 & 0.1991 & 0.2308 & 0.1956 & 0.1726 & 0.2003 & 0.1477 & 0.1699 \\
\hline & 0.0172 & 0.0172 & 0.0173 & 0.0174 & $\begin{array}{c}(* * *) \\
0.0170\end{array}$ & $\begin{array}{c}(* * *) \\
0.0172\end{array}$ & $\begin{array}{c}(* * *) \\
0.0172\end{array}$ & $\begin{array}{c}(* * *) \\
0.0181\end{array}$ & $\begin{array}{c}(* * *) \\
0.0183\end{array}$ & $\begin{array}{c}(* * *) \\
0.0183\end{array}$ \\
\hline \multirow{2}{*}{$\begin{array}{l}\text { Vive } \\
\text { Solo }\end{array}$} & 0.1388 & 0.0584 & 0.2073 & 0.1975 & 0.2482 & 0.2226 & 0.2519 & 0.2780 & 0.2309 & 0.2777 \\
\hline & $\begin{array}{c}(* * *) \\
0.0301\end{array}$ & $\begin{array}{c}(* *) \\
0.0257\end{array}$ & $\begin{array}{c}(* * *) \\
0.0287\end{array}$ & $\begin{array}{c}(* * *) \\
0.0292\end{array}$ & $\begin{array}{c}(* * *) \\
0.0288\end{array}$ & $\begin{array}{c}(* * *) \\
0.0278\end{array}$ & $\begin{array}{c}(* * *) \\
0.0275\end{array}$ & $\begin{array}{c}(* * *) \\
0.0291\end{array}$ & $\begin{array}{c}(* * *) \\
0.0277\end{array}$ & $\begin{array}{r}(* * *) \\
0.0274\end{array}$ \\
\hline \multirow{2}{*}{ Mujer } & 0.1499 & 0.1395 & 0.1165 & 0.1510 & 0.1484 & 0.1436 & 0.1499 & 0.1628 & 0.1496 & 0.1646 \\
\hline & 0.0145 & 0.0144 & 0.0145 & 0.0144 & 0.0141 & 0.0141 & 0.0141 & 0.0149 & 0.0148 & 0.0148 \\
\hline \multirow{2}{*}{ Viudo } & $\begin{array}{c}0.1390 \\
(* * *)\end{array}$ & $\begin{array}{c}0.1363 \\
(* * *)\end{array}$ & 0.0533 & $\begin{array}{c}0.1393 \\
(* * *)\end{array}$ & $\begin{array}{c}0.0814 \\
(* * *)\end{array}$ & $\begin{array}{c}0.1486 \\
(* *)\end{array}$ & $\begin{array}{c}0.1401 \\
(* * *)\end{array}$ & 0.1721 & $\begin{array}{c}0.0825 \\
(* * *)\end{array}$ & $\begin{array}{c}0.0765 \\
(* *)\end{array}$ \\
\hline & 0.0301 & 0.0294 & 0.0291 & 0.0296 & 0.0289 & 0.0290 & 0.0289 & 0.0314 & 0.0305 & 0.0306 \\
\hline \multirow{2}{*}{ Divorciado } & 0.0648 & 0.1143 & 0.0779 & 0.1120 & 0.0392 & 0.1293 & 0.1394 & 0.1252 & 0.0817 & 0.0917 \\
\hline & 0.0323 & 0.0321 & 0.0323 & 0.0324 & 0.0300 & 0.0304 & 0.0300 & 0.0318 & 0.0310 & 0.0309 \\
\hline \multirow{2}{*}{ Casado } & $\begin{array}{c}0.0666 \\
(* * *)\end{array}$ & $\begin{array}{c}0.0548 \\
(* * *)\end{array}$ & 0.0055 & $\begin{array}{c}0.0630 \\
(* * *)\end{array}$ & 0.0181 & $\begin{array}{c}0.0893 \\
(* *)\end{array}$ & $\begin{array}{c}0.1333 \\
(* * *)\end{array}$ & $\begin{array}{c}0.1406 \\
(* * *)\end{array}$ & 0.0512 & $\begin{array}{c}0.0800 \\
(* * *)\end{array}$ \\
\hline & 0.0271 & 0.0205 & 0.0207 & 0.0208 & 0.0199 & 0.0204 & 0.0201 & 0.0215 & 0.0211 & 0.0213 \\
\hline \multirow{2}{*}{ Educación } & -0.0296 & -0.0541 & -0.0171 & -0.0091 & -0.0287 & -0.0437 & -0.0097 & -0.0207 & -0.0053 & -0.0252 \\
\hline & 0.0059 & 0.0059 & 0.0060 & 0.0060 & 0.0059 & $\begin{array}{l}(5.6) \\
0.0060\end{array}$ & 0.0060 & 0.0065 & 0.0064 & 0.0066 \\
\hline \multirow{2}{*}{ Educación ${ }^{2}$} & 0.0014 & 0.0023 & 0.0006 & 0.0002 & 0.0014 & 0.0021 & 0.0007 & 0.0012 & 0.00007 & 0.0015 \\
\hline & $\begin{array}{c}(* * *) \\
0.0003\end{array}$ & $\begin{array}{c}(* * *) \\
0.0003\end{array}$ & 0.0003 & 0.0003 & 0.0003 & 0.0003 & $\begin{array}{c}(* *) \\
0.0003\end{array}$ & $\begin{array}{c}(* * *) \\
0.0003\end{array}$ & $\begin{array}{c}(* *) \\
0.0003\end{array}$ & $\begin{array}{l}(* * *) \\
0.0003\end{array}$ \\
\hline \multirow[b]{2}{*}{ Constante } & -1.2876 & -1.0747 & -1.3847 & -1.4649 & -1.3597 & -1.3847 & -1.4737 & -1.3980 & -1.4980 & -1.5337 \\
\hline & $\begin{array}{l}(* * *) \\
0.0454\end{array}$ & $\begin{array}{c}(* * *) \\
0.0448\end{array}$ & $\begin{array}{c}(* * *) \\
0.0473\end{array}$ & $\begin{array}{c}(* * *) \\
0.0473\end{array}$ & $\begin{array}{c}(* * *) \\
0.0450\end{array}$ & $\begin{array}{c}(* * *) \\
0.0481\end{array}$ & $\begin{array}{c}(* * *) \\
0.0471\end{array}$ & $\begin{array}{l}(* * *) \\
0.0518\end{array}$ & $\begin{array}{c}(* * *) \\
0.0526\end{array}$ & $\begin{array}{l}(* * *) \\
0.0555\end{array}$ \\
\hline $\mathrm{R}^{2}(\%)$ & 6.77 & 7.08 & 9.23 & 9.01 & 8.98 & 7.92 & 6.70 & 6.56 & 6.47 & 7.80 \\
\hline
\end{tabular}

Nota: (***) Significativo al 1\%; (**) Significativo al 5\%; (*) Significativo al $10 \%$. 


\section{CUADRO 6}

EFECTOS MARGINALES. ESTIMACIÓN PROBIT. VARIABLE DEPENDIENTE: ESTADO DE SALUD ( $1=$ MAL ESTADO, 0= BUEN ESTADO)

(Población de 18 y más años de edad. Puntos porcentuales)

\begin{tabular}{|l|r|r|r|r|r|r|r|r|r|r|}
\hline Año & 1991 & 1992 & 1993 & 1994 & 1995 & 1996 & 1997 & 1998 & 1999 & 2000 \\
\hline Edad 26-35 & 4.71 & 2.47 & 2.68 & 2.97 & 6.09 & 1.66 & - & 1.61 & & \\
Edad 36-45 & 7.62 & 4.14 & 7.74 & 6.83 & 8.43 & 3.62 & 4.55 & - & 4.18 & 3.09 \\
Edad 46-59 & 14.38 & 11.39 & 15.56 & 14.96 & 14.54 & 11.53 & 10.51 & 8.91 & 10.18 & 9.71 \\
Edad 60+ & 25.69 & 22.40 & 29.50 & 27.34 & 28.76 & 22.27 & 22.21 & 19.28 & 22.41 & 21.98 \\
Pública Mvd & 14.95 & 13.70 & 18.11 & 23.35 & 24.66 & 23.45 & 17.16 & 9.42 & 7.26 & 9.73 \\
Pública Interior & 13.03 & 10.84 & 13.21 & 10.53 & 12.05 & 13.99 & 12.07 & 13.95 & 15.44 & 18.45 \\
Privada Mvd & 17.04 & 18.19 & 19.45 & 24.66 & 24.81 & 25.30 & 19.07 & 11.61 & 11.02 & 13.69 \\
Privada Interior & 19.37 & 15.49 & 17.88 & 13.24 & 15.82 & 19.68 & 19.51 & 18.40 & 20.97 & 27.08 \\
Desocupado & 3.21 & 4.26 & 5.14 & 4.83 & 4.60 & 4.19 & 3.66 & 5.45 & 4.12 & 2.59 \\
Inactivo & 7.06 & 7.99 & 8.91 & 7.18 & 8.53 & 6.93 & 6.10 & 6.80 & 4.86 & 5.54 \\
Solo & 5.16 & 2.14 & 7.70 & 7.31 & 9.43 & 8.14 & 9.25 & 9.89 & 7.95 & 9.57 \\
Mujer & 5.42 & 5.06 & 4.16 & 5.37 & 5.41 & 5.01 & 5.23 & 5.42 & 4.84 & 5.27 \\
Viudo & 5.16 & 5.06 & 1.93 & 5.09 & 3.02 & 5.36 & 5.04 & 5.98 & 2.74 & 2.51 \\
Divorciado & 2.38 & 4.24 & 2.83 & 4.09 & - & 4.65 & 5.02 & 4.31 & 2.72 & 3.03 \\
Casado & 2.41 & 1.99 & - & 2.24 & - & 3.12 & 4.64 & 4.66 & 1.66 & 2.56 \\
Educación & -1.07 & -1.97 & -0.61 & - & -1.44 & -1.53 & - & -0.69 & - & -0.81 \\
Educación ${ }^{2}$ & 0.05 & 0.09 & 0.02 & - & 0.66 & 0.07 & 0.03 & 0.04 & 0.02 & 0.05 \\
\hline
\end{tabular}

Fuente: Elaboración propia en base a las ECH 1991-2000, INE.

Nota: Los efectos marginales fueron calculados con el comando "mfx" de Stata/SE8 (StataCorp, 2005). En el caso que las variables independientes sean binarias el efecto marginal es el cambio en la probabilidad de pasar de 0 a 1 , mientras que si la variable es continua el efecto marginal es el impacto en la probabilidad de un cambio marginal en la media de la variable. Se presentan los efectos marginales únicamente de las variables significativas al $10 \%$ o más.

\section{CUADRO 7}

PROBABILIDAD DE UN MAL ESTADO DE SALUD PARA GRUPOS ESPECÍFICOS. ESTIMACIÓN PROBIT. VARIABLE DEPENDIENTE: ESTADO DE SALUD (1= MAL ESTADO, $0=$ BUEN ESTADO)

(Población de 18 y más años de edad)

\begin{tabular}{|l|c|c|c|c|c|c|c|c|c|c|}
\hline Año & 1991 & 1992 & 1993 & 1994 & 1995 & 1996 & 1997 & 1998 & 1999 & 2000 \\
\hline Prob. Prom. & 0.3322 & 0.3334 & 0.3207 & 0.3192 & 0.3393 & 0.3055 & 0.3048 & 0.2764 & 0.2616 & 0.2563 \\
Edad 18-25 & 0.2233 & 0.2429 & 0.2036 & 0.2096 & 0.2191 & 0.2200 & 0.2195 & 0.2107 & 0.1833 & 0.1809 \\
Edad 26-35 & 0.2632 & 0.2644 & 0.2252 & 0.2340 & 0.2703 & 0.2341 & - & 0.2248 & - & - \\
Edad 36-45 & 0.2890 & 0.2793 & 0.2649 & 0.2671 & 0.2911 & 0.2512 & 0.2590 & - & 0.2184 & 0.2067 \\
Edad 46-59 & 0.3523 & 0.3471 & 0.3400 & 0.3422 & 0.3477 & 0.3242 & 0.3140 & 0.2918 & 0.2725 & 0.2659 \\
Edad 60 y+ & 0.4684 & 0.4597 & 0.4853 & 0.4705 & 0.4941 & 0.4345 & 0.4338 & 0.3974 & 0.3974 & 0.3912 \\
Ocupado & 0.3038 & 0.3009 & 0.2842 & 0.2899 & 0.3054 & 0.2770 & 0.2795 & 0.2496 & 0.2417 & 0.2348 \\
Desocupado & 0.3348 & 0.3419 & 0.3333 & 0.3365 & 0.3499 & 0.3174 & 0.3149 & 0.3019 & 0.2816 & 0.2597 \\
Inactivo & 0.3743 & 0.3806 & 0.3730 & 0.3614 & 0.3905 & 0.3460 & 0.3403 & 0.3172 & 0.2901 & 0.2900 \\
Solo & 0.3809 & 0.3532 & 0.3930 & 0.3879 & 0.4281 & 0.3819 & 0.3915 & 0.3691 & 0.3356 & 0.3452 \\
Mujer & 0.3574 & 0.3565 & 0.3396 & 0.3440 & 0.3642 & 0.3286 & 0.3291 & 0.3018 & 0.2842 & 0.2809 \\
Soltero & 0.3110 & 0.3137 & 0.3158 & 0.2983 & 0.3313 & 0.2791 & 0.2698 & 0.2409 & 0.2473 & 0.2369 \\
Divorciado & 0.3342 & 0.3553 & 0.3440 & 0.3382 & - & 0.3241 & 0.3178 & 0.2815 & 0.2738 & 0.2661 \\
Viudo & 0.3616 & 0.3635 & 0.3350 & 0.3482 & 0.3613 & 0.3311 & 0.3180 & 0.2976 & 0.2741 & 0.2612 \\
Casado & 0.3349 & 0.3334 & - & 0.3205 & - & 0.3099 & 0.3156 & 0.2867 & 0.2638 & 0.2623 \\
Educación 0 años & 0.4188 & 0.4964 & 0.3703 & - & 0.4273 & 0.4397 & - & 0.3371 & - & 0.3313 \\
Educación 20 años & 0.2129 & 0.1379 & 0.2506 & - & 0.2244 & 0.1524 & - & 0.2018 & - & 0.1734 \\
\hline
\end{tabular}

Fuente: Elaboración propia en base a las ECH 1991-2000, INE.

Nota: No se calcularon las probabilidades para los casos en que las variables no son significativas al $10 \%$. 


\section{GRÁFICO 1 \\ PROBABILIDAD PREDICHA DE REPORTAR UN \\ MAL ESTADO DE SALUD}

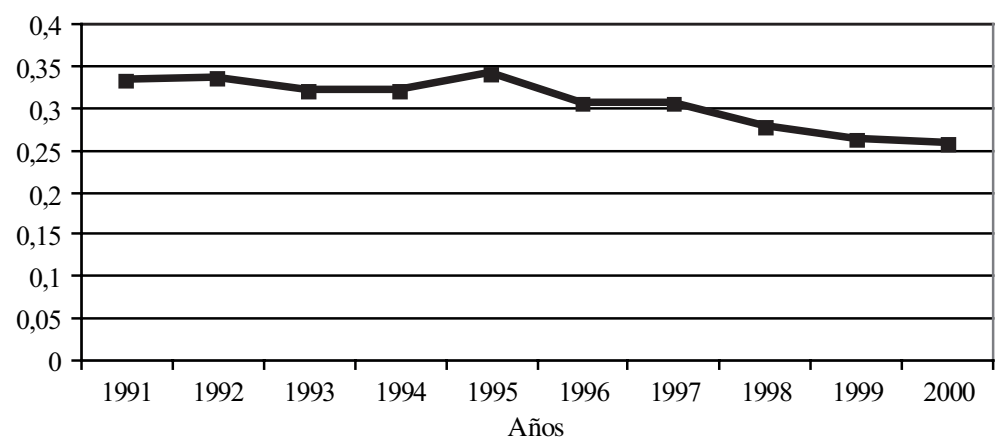

Fuente: Elaboración propia en base a información de la ECH, 1991-2000, INE.

El hecho de estar desocupado tiene un efecto marginal en torno a los 3 puntos porcentuales. Por su parte, la condición de inactivo tiene un impacto mayor, que se ubica alrededor de los 5 puntos porcentuales. Adicionalmente, se estudia la probabilidad de un mal estado de salud para cada condición de actividad, encontrándose que los inactivos tienen, en el período analizado, la mayor probabilidad de tener un mal estado de salud. Este resultado es esperable, puesto que dentro de dicho grupo los jubilados y pensionistas son la mayoría y, en general, pertenecen al tramo etario de 60 años y más, caracterizado por tener la mayor probabilidad de un mal estado de salud. No obstante, la significación de dicha variable indicaría que existen otros efectos no vinculados a la edad.

Por otro lado, se constata que los solteros tienen un mejor estado de salud que los individuos pertenecientes a las restantes categorías de estado civil. No obstante, la categoría casado o conviviente no resulta significativa para los años 1993 y 1995. Asimismo, el impacto observado es moderado en todos los casos, no siendo posible ordenar las categorías casado, divorciado y viudo en función de sus efectos marginales, puesto que las posiciones relativas varían en el período.

Este resultado diverge respecto a lo hallado por otros autores, en particular para países desarrollados. En este sentido, Gerdtham y Johasnnesson (1997) y Gardner y Oswald (2004) encuentran un efecto positivo del matrimonio en el buen estado de salud y el aumento de la longevidad en países desarrollados, al tiempo que Oswald y Wilson (2005) realizan una revisión de la evidencia entre estado de salud y matrimonio, encontrando que el impacto de este último sobre la salud puede ser tan importante como dejar de fumar.

No obstante, Jewell et al. (2006), al analizar el estado de salud de los adultos mayores en América Latina, encuentran que aquellos individuos uruguayos que nunca estuvieron casados o en unión libre tienen una probabilidad mayor de reportar un buen estado de salud, al tiempo que esta variable no resulta significativa en los demás países analizados (Argentina, Brasil, Chile y México). Este hallazgo podría estar confirmando un comportamiento atípico de la correlación entre estado de salud y estado civil en el caso uruguayo. 
Más allá del estado civil del individuo, interesa estudiar los efectos del hecho de vivir solo, observándose una correlación positiva con el mal estado de salud, en concordancia con la evidencia relevada en otros países. Asimismo, con excepción de los dos primeros años, el impacto de dicha variable es considerable, situándose entre 7 y 10 puntos porcentuales, siendo bastante más importante que el efecto encontrado para las distintas categorías de estado civil. Podrían estar jugando en este caso los efectos de las redes familiares, en especial para el caso de los jóvenes (Jewell et al. (2005)), así como las limitaciones funcionales, en particular en las edades avanzadas.

Como era de esperarse, existe una asociación positiva entre el hecho de ser mujer y el estado de salud, siendo este impacto prácticamente constante a lo largo de la década analizada. Sin embargo, el efecto encontrado es moderado, oscilando en torno a los 5 puntos porcentuales. Esto podría reflejar que la mayor esperanza de vida de la mujer implica un mayor tiempo de padecimiento de condiciones crónicas. Por otro lado, el umbral de autopercepción de estado de salud podría ser distinto entre sexos, así como el comportamiento en lo que refiere al uso de cuidados médicos.

Cabe destacar que en varios estudios aplicados a países latinoamericanos, se encuentra esta relación entre el sexo y el estado de salud. ${ }^{4}$ No obstante, la misma suele invertirse al estudiar países desarrollados. Dada la mayor esperanza de vida de las mujeres tanto en países desarrollados como en países en vías de desarrollo, este resultado podría estar indicando una mejor atención sanitaria, económica, y de seguridad social en general, disponible para las mujeres en los países desarrollados, sobre todo a edades avanzadas.

En relación al impacto del aumento en los años de educación, se tiene que cada año de instrucción formal adicional reduce la probabilidad de reportar un mal estado de salud en alrededor de 1 punto porcentual. En este sentido, si bien el impacto resulta moderado y no significativo para los años 1994, 1997 y 1999, el signo es el esperado, asemejando los resultados obtenidos a los hallados en todos los estudios consultados. Esto podría deberse a que aquellos con mayor nivel educativo utilizarían en forma más eficiente sus conocimientos y los recursos disponibles.

Se debe tener en cuenta que, debido a que la pregunta formulada en la ECH indaga acerca de la ocurrencia de enfermedad y no del estado de salud general, dichos coeficientes podrían tener un sesgo hacia abajo. En este sentido, los individuos con mayor nivel educativo tienen un mayor conocimiento acerca de las enfermedades y, por lo tanto, tenderían a reportarlas en mayor medida, que aquellos con menor nivel de instrucción.

Por otra parte, se observa que los coeficientes de los años de educación al cuadrado son negativos, lo que implica que la probabilidad de reportar un mal estado de salud decrece con los años de educación hasta cierto umbral para luego invertirse esta relación. Cabe destacar que el punto de inflexión parece haberse visto afectado por el cambio en la muestra acontecido en la ECH del año 1998 (ver Gráfico 2). En este sentido, antes del cambio muestral, dicho

4 Véase por ejemplo Jewell et al. (2006), donde el hecho de ser mujer tiene un impacto negativo y significativo en la probabilidad de reportar un mejor estado de salud en Argentina, Brasil, Chile, México y Uruguay. 
GRÁFICO 2

PUNTO DE INFLEXIÓN DE LOS AÑOS DE EDUCACIÓN

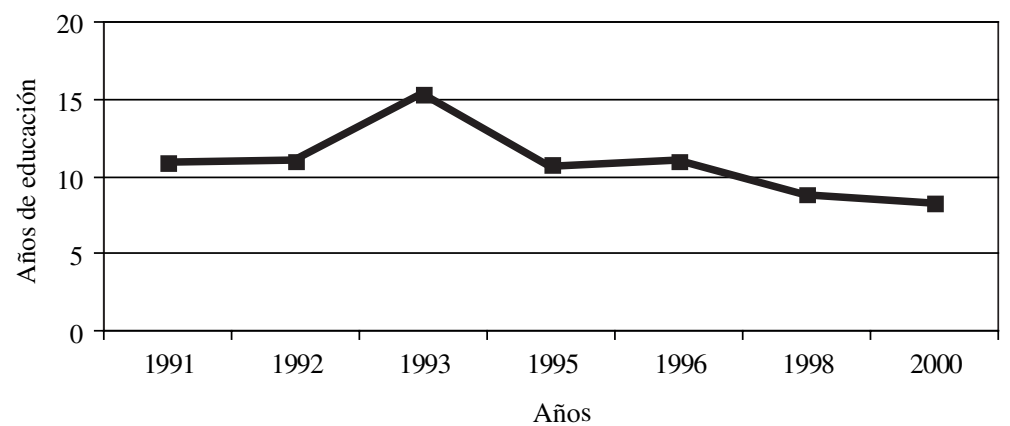

Fuente: Elaboración propia en base a las ECH 1991-2000, INE.

Nota: Calculada para los años en que la variable Educación resulta significativa al menos al $1 \%$.

umbral se encontraba en torno a los 10 años de instrucción; mientras que luego del cambio se encuentra alrededor de los 8 años de educación formal. ${ }^{5}$

Respecto a la cobertura de salud, se encuentra una correlación positiva entre cobertura y probabilidad de que el individuo reporte un mal estado. Cabe destacar que los individuos sin cobertura suelen ser de estatus socioeconómico bajo, pudiendo tener menos conocimientos acerca de su estado de salud general. Por otra parte, quienes se perciben como enfermos pueden tender a asegurarse más y mejor.

El análisis de la probabilidad de consultar por tipo de cobertura, dado que el individuo efectivamente se ha sentido enfermo, muestra que el tener cobertura de salud tiene impactos positivos y significativos al $99 \%$ de confianza en la probabilidad de consultar, en todos los años analizados. Para Montevideo, el impacto sobre dicha probabilidad es mayor para quienes tienen cobertura privada que pública, respecto de los que no tienen cobertura. Cabe recordar que en la decisión de consultar juegan una serie de costos monetarios adicionales, como tiques, órdenes, etc; y no monetarios, como ser traslados, colas, demoras, etc. Estos últimos son mayores en el caso de la cobertura pública, en especial para Montevideo. Adicionalmente, quienes tienen este tipo de cobertura suelen ser de un estatus socioeconómico más bajo que quienes tienen cobertura privada. Esto puede explicar el hecho de que quienes tienen cobertura pública tengan una probabilidad menor de consultar que quienes tienen cobertura privada en Montevideo, siempre evaluadas respecto a aquellos sin cobertura. En el interior, se suele percibir una calidad superior de los servicios públicos en comparación con Montevideo, lo cual podría explicar que no exista una diferencia tan marcada entre los impactos de los diferentes tipos de cobertura en el interior. Ver Cuadro 8.

5 Este resultado se asemeja al obtenido por Grignon (2005) en su análisis de 111 países, desarrollados y subdesarrollados. 
Finalmente, resulta interesante analizar la probabilidad de tener un mal estado de salud para grupos específicos, como ser el más riesgoso (tiene todas las características que determinan un mal estado de salud), y el menos riesgoso (no tiene ninguna de dichas características), comparándolo a su vez con el promedio. En este sentido, se calcula la probabilidad para el grupo menos riesgoso: un hombre, soltero, de 18 a 25 años de edad, que no vive solo, ocupado, y con 20 años de educación formal. Por otra parte, se realiza el mismo cálculo para el grupo más riesgoso: una mujer, viuda, de 60 o más años de edad, que vive sola, inactiva, y sin instrucción formal (ver Gráfico 3).

Se observa que la probabilidad de tener un mal estado de salud se ve reducida en tres veces respecto a la probabilidad promedio, cuando se la calcula para un individuo con todas las características que contribuyen a un buen estado de salud. Por su parte, la probabilidad de tener un mal estado de salud calculada para una persona con todos los factores que afectan negativamente

\author{
CUADRO 8 \\ EFECTOS MARGINALES DE LA COBERTURA DE SALUD. \\ VARIABLE DEPENDIENTE: CONSULTA (=1 SI EL INDIVIDUO REALIZÓ \\ UNA CONSULTA MÉDICA, DADO QUE SE SINTIÓ ENFERMO) \\ (Población de 18 años y más. Puntos porcentuales)
}

\begin{tabular}{|lrrrrrrrrrr|}
\hline & 1991 & 1992 & 1993 & 1994 & 1995 & 1996 & 1997 & 1998 & 1999 & 2000 \\
\hline Pública Montevideo & 5.64 & 10.08 & 6.42 & 2.56 & 2.92 & 3.81 & 6.45 & 5.13 & 5.44 & 3.93 \\
Pública Interior & 7.73 & 3.77 & 2.87 & 5.60 & 3.49 & 5.75 & 10.29 & 12.00 & 8.55 & 5.85 \\
Privada Montevideo & 9.11 & 16.40 & 11.01 & 5.27 & 4.12 & 4.98 & 7.37 & 6.39 & 6.95 & 5.14 \\
Privada Interior & 10.42 & 8.02 & 4.92 & 6.31 & 3.95 & 5.46 & 10.55 & 10.63 & 8.27 & 6.10 \\
\hline
\end{tabular}

Fuente: Elaboración propia en base a las ECH 1991-2000, INE.

Nota: Los efectos marginales fueron calculados con el comando "mfx" de Stata/SE8 (ver StataCorp (2005). El efecto marginal corresponde al impacto de las variables independientes en el cambio de la variable dependiente de 0 a 1 (variables independientes binarias), respecto de quienes no tienen cobertura de salud.Se presentan los efectos marginales únicamente de las variables significativas al $10 \%$ o más.

GRÁFICO 3

PROBABILIDAD DE REPORTAR UN MAL ESTADO DE SALUD

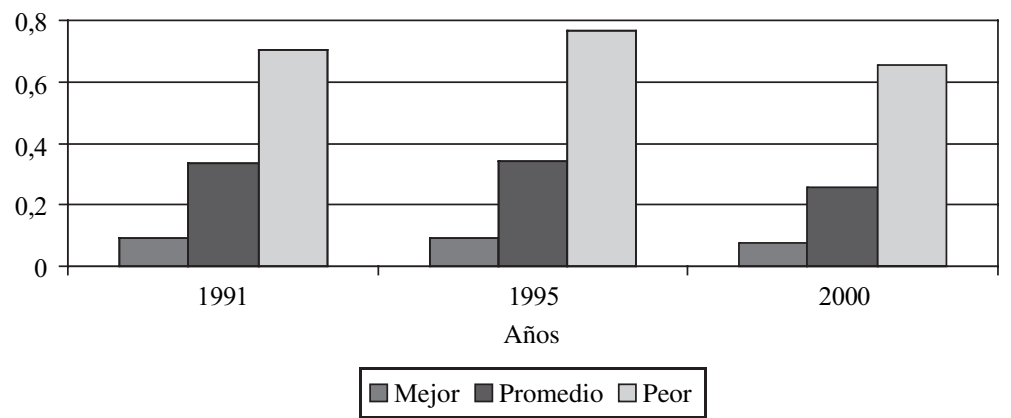

Fuente: Elaboración propia en base a las ECH 1991, 1995 y 2000, INE. 
la salud, más que duplica la probabilidad promedio, y es 8 veces la del primer grupo.

En este tipo de modelos se suelen encontrar bajas medidas de bondad de ajuste (Pseudo R2). ${ }^{6}$ Sin embargo, de acuerdo a Long y Freese (2001), éstas proveen solamente información parcial que debe ser considerada en el contexto de la teoría que motiva el análisis, la investigación pasada y los parámetros estimados en el modelo que se está considerando. Por lo tanto, se considera que una medida adecuada es la capacidad de predecir correctamente el buen y mal estado de salud. La tasa de aciertos corresponde a la suma de ceros (buen estado) y unos (mal estado) correctamente predichos en relación al número de observaciones totales (ver Cuadro 9).

En términos generales, puede decirse que el modelo tiene un buen poder predictivo, pues la tasa de aciertos totales gira en torno a un $65 \%$. No obstante, debe tenerse en cuenta que el modelo predice mejor el buen estado de salud (0) que el malo (1). El porcentaje de ceros correctamente predichos oscila entre el $75 \%$ y el $82 \%$ durante el período; mientras que el de unos varía entre un $40 \%$ y un $50 \%$. Cabe recordar que no se dispone de información acerca de los hábitos de riesgo de la población, como ser fumar, beber, sedentarismo, etc., ni de la calidad de las redes familiares y otros elementos vinculados al capital social, factores que tienen una fuerte incidencia sobre el estado de salud de los individuos.

Dados los hallazgos respecto a la edad, parece relevante realizar un análisis complementario para edades más homogéneas no extremas, debido a que las funciones de producción de salud podrían ser diferentes a distintas edades. En este sentido, se realizaron las estimaciones para el tramo etario de los individuos entre 26 y 35 años de edad. ${ }^{7}$

Cabe destacar que existen diferencias sustanciales en las estimaciones realizadas para este tramo etario respecto a las estimaciones generales. Respecto a

\section{CUADRO 9}

BONDAD DE AJUSTE DE MODELO. PORCENTAJE DE 0 Y 1 CORRECTAMENTE PREDICHOS POR EL MODELO, Y PORCENTAJE DE ACIERTOS TOTALES. ESTIMACIÓN PROBIT. VARIABLE DEPENDIENTE: ESTADO DE SALUD $(1=$ MAL ESTADO, $0=$ BUEN ESTADO $)$

(Población de 18 y más años de edad)

\begin{tabular}{|ccccccccccc|}
\hline & 1991 & 1992 & 1993 & 1994 & 1995 & 1996 & 1997 & 1998 & 1999 & 2000 \\
\hline 0 & 75.74 & 75.94 & 78.00 & 78.08 & 75.82 & 78.35 & 77.71 & 79.67 & 80.96 & 81.94 \\
1 & 48.21 & 49.64 & 50.96 & 48.74 & 52.04 & 47.01 & 45.25 & 42.24 & 39.84 & 40.48 \\
Aciertos & 64.25 & 65.33 & 66.60 & 65.52 & 66.41 & 65.61 & 64.45 & 64.91 & 63.94 & 64.81 \\
\hline
\end{tabular}

Fuente: Elaboración propia en base a las ECH 1991-2000, INE.

$6 \quad$ En general los pseudos $\mathrm{R}^{2}$ de este tipo de modelos se encuentran entre $3 \%$ y $12 \%$ de acuerdo a Wallace y Guitierrez (2005), y Gerdtham y Johanneson (1997), entre otros.

7 Los resultados de las estimaciones se encuentran disponibles por los autores para los interesados. 
la condición de ocupación, se observa que el estar desocupado es significativo sólo para los años 1998 y 2000, con efectos marginales un tanto mayores a los estimados en el modelo general ( 7 y 8 puntos porcentuales). Asimismo, el ser inactivo es significativo en todos los casos, con efectos marginales un tanto menores. Cabe destacar que la composición de los inactivos en el total de la población mayor de 18 años y en el caso de los individuos de entre 26 y 35 años es diferente. En el primer caso, el $60 \%$ son jubilados y pensionistas, mientras que en el segundo caso, el $58 \%$ son amas de casa. Este hallazgo podría estar reflejando la importancia de las redes sociales laborales de los individuos.

Respecto al estado civil, cuando la variable estar casado es significativa al menos al 5\% (años 1995, 1996 y 2000), se comporta exactamente igual al modelo general, con efectos marginales similares. No obstante, en la mayoría de los años analizados, tanto esta variable como el hecho de estar divorciado o separado no son significativas.

Por otra parte, se observa que el hecho de ser mujer y la condición de vivir solo tienen el mismo impacto que en el modelo general, con efectos marginales similares (salvo que el vivir solo no resulta significativo en los años 1992, 1996 y 1998). Este hallazgo reafirma lo dicho respecto a las redes sociales y a la mejor atención brindada a la mujer en los países desarrollados, en particular en términos de seguridad social en general.

Por último, en relación a la educación, se observa que dicha variable no resulta significativa en ningún caso. Cabe destacar que en el modelo general se estimó que la probabilidad de reportar un mal estado de salud era decreciente con los años de educación hasta alcanzar el umbral de 8 años de instrucción formal. En este sentido, cabe notar que en la población más joven, los niveles educativos suelen ser más altos y, en el caso particular de los individuos entre 26 y 35 años de edad, la media de los años de educación se encuentra entre 9.5 y 10.1 en los 10 años analizados.

\section{Conclusiones}

En el presente trabajo se estudia uno de los componentes del capital humano, quizás el menos estudiado, la salud. A menudo se quiere hacer inferencia causal acerca de diferentes variables sobre la misma. Sin embargo, hacerlo en escenarios no experimentales es frecuentemente dificultoso, dado que algunas de las variables explicativas son endógenas; esto es, son causadas por algunas de las mismas fuerzas que influencian el problema bajo estudio. La imposibilidad de contar con adecuados instrumentos que permitieran probar la existencia de endogeneidad, y en ese caso controlarla, lleva a que únicamente se puedan detectar correlaciones entre las diferentes variables y el capital salud, dando indicios de grupos de riesgo, para los cuales se deberían profundizar los estudios a efecto del diseño de política.

A través de la utilización de las Encuestas Continuas de Hogares $(\mathrm{ECH})$ de Uruguay de 1991 a 2000, se estiman modelos probit, encontrando que tienen una menor probabilidad de declarar un mal estado de salud los hombres, los solteros, quienes no viven solos, los más jóvenes y los ocupados, al tiempo que dicha probabilidad se ve reducida con los años de instrucción formal. Asimismo, al evaluar la evolución del estado de salud de los individuos en el período 
considerado, se encuentra que la probabilidad de tener un mal estado de salud presenta una tendencia descendente, pasando de 0.33 en el año 1991 a 0.25 en el año 2000. En general, para el decenio analizado los resultados muestran un alto poder predictivo del modelo respecto del estado de salud, con una tasa de aciertos en torno al $65 \%$.

En lo que respecta al sexo, los hallazgos pueden estar reflejando que la mayor esperanza de vida de las mujeres da lugar a un padecimiento de condiciones crónicas durante un período más prolongado en países en vías de desarrollo, o que las mismas tienen un umbral en la autopercepción de su estado de salud diferente a los hombres, llevándolas a una mayor tasa de utilización de cuidados médicos. La evidencia empírica disponible para países desarrollados no verifica este hallazgo, lo que podría estar reflejando una mejor atención sanitaria, económica, y de seguridad social en general para las mujeres de estos países.

Por su parte, se encuentra una asociación positiva entre el mal estado de salud y la condición de vivir solo, lo cual podría estar recogiendo deficiencias de capital social del individuo, como carencia de vínculos afectivos que le brinden seguridad y estabilidad emocional, entre otras.

En relación al estado civil, se encuentra una asociación positiva entre el ser casado y reportar un mal estado de salud. Este hallazgo constituye una anomalía si lo comparamos con las vinculaciones entre el matrimonio y la salud encontradas a nivel internacional. Sería razonable pensar que el matrimonio, en tanto vínculo afectivo y social, incrementa el capital social del individuo, con lo que su impacto en el buen estado de salud sería positivo. Sin embargo, otros estudios aplicados a Uruguay, utilizado otras fuentes de información encuentran el mismo efecto que en el presente estudio.

Respecto a la edad, lo encontrado es consistente con la teoría, en el sentido de que la misma incrementaría la depreciación del stock de salud de los individuos. En este sentido, se encuentra que el grupo formado por los individuos de 60 y más años tienen la mayor probabilidad de tener un mal estado de salud.

Al analizar la condición de actividad, se encuentra una asociación positiva entre el mal estado de salud y encontrarse desocupado o ser inactivo. Este último grupo está formado por una alta proporción de jubilados y pensionistas, y por lo tanto por individuos de 60 años y más, por lo que dicha variable estaría recogiendo otras características no atribuibles a la edad que implican la condición de actividad, como ser el sedentarismo, las redes sociales, etc.

En lo que se refiere al nivel educativo, los resultados muestran una correlación negativa entre éste y el mal de salud, pudiéndose a su vez utilizarlo como un indicador del estatus socioeconómico (nivel de ingreso, acceso y procesamiento de la información, etc). Sin embargo, los efectos marginales son moderados y se observa un punto de inflexión alrededor de los 8 años de educación, asemejándose a los resultados hallados para varios países desarrollados y subdesarrollados.

No obstante, estos resultados pueden estar influidos por el hecho de que en las ECH se indaga acerca de la ocurrencia de enfermedad en los últimos 12 meses y no de la autopercepción del estado de salud general. En este sentido, los individuos con mayor nivel educativo podrían tener un mejor conocimiento acerca de las enfermedades y podrían tender a reportarlas en mayor medida, que aquellos con menor nivel de instrucción, reduciendo el impacto del incremento en el nivel educativo. 
Por último, por tipo de cobertura médica se encuentra una asociación negativa entre no tener ningún tipo de cobertura y mal estado de salud, lo cual podría explicarse por el hecho de que estos individuos suelen ser de estatus socioeconómico bajo, pudiendo tener menos conocimientos acerca de las enfermedades. Adicionalmente, quienes se perciben como enfermos es esperable que tiendan a asegurarse más y mejor. Con el objetivo de verificar esto último, se realizó un análisis probit para determinar la probabilidad de que un individuo realice una consulta médica, dado que se sintió enfermo; encontrándose una relación positiva entre dicha probabilidad y la cobertura médica, siendo mayor para quienes tienen un seguro privado.

\section{Bibliografía}

Baker, M., M. Stabile y C. Deri (2001). "What do self-reported, objective, measures of health measure?" Working Paper 8419, National Bureau of Economic Research.

Benítez-Silva, H., M. Buchinsky, H.M. Chan, S. Cheidvasser y J. Rust (2002). "How large is the bias in self-reported disability?" Working Paper 7526, National Bureau of Economic Research.

Bound, J. (1991). "Self-reported versus objective measures of health in retirement models", The Journal of Human Resources, Vol. 26; 106-38.

Bucheli, M. y M. Furtado (2001). "Efectos de los cambios en el relevamiento de las Encuestas Continuas de Hogares sobre los principales indicadores socioeconómicos", LC/MVD/R.188, Oficina de la CEPAL, Montevideo.

Connelly, J.E., J.T. Philbrick, R. Smith, D.L. Kaiser, y A. Wymer (1989). "Health perceptions of primary care patients and the influence on health care utilization", Supplement to Medical Care, Vol. 27; 99-109.

Costa, D. y J. Lahey (2003). "Becoming Oldest-Old: Evidence from Historical U.S. Data”, Working Paper 9933, National Bureau of Economic Research.

Deaton, A. (2001). "Health, inequality and economic development", Working Paper 8318, National Bureau of Economic Research.

Fogel, R.W. (2004). "Changes in the Disparities in Chronic Disease During the Course of the Twentieth Century", Working Paper 10311, National Bureau of Economic Research.

Gardner, J. y A.J. Oswald (2004). "How is Mortality Affected by Money, Marriage and Stress?", Journal of Health Economics, Vol. 23; 1181-1207.

Gerdtham, U. y M. Johannesson (1997). "New estimates of the demand for health: results based on a categorical health measure and Swedish micro data”, Working Paper 205, Working Paper Series in Economics and Finance, Stockholm School of Economics.

Greene, W.H. (1981). "Sample selection bias as a specification error: comment", Econometrica, Vol. 49, 795-798.

Grignon, M. (2005). "The role of education in health system performance. A Propos World Health Report 2000", Center for Health Economics and Policy Analysis (CHEPA) Working Paper Series, Paper 06-03, McMaster University, Ontario. 
Grossman, M. (2003). "The human capital model”, En: Handbook of health economics, Vol. 1A, cap. 7; 347-405. Editores: Culyer, A. y J. Newhouse.

Grossman, M. (1972a). "The demand for health: A theoretical and empirical investigation", Columbia University Press for the National Bureau of Economic Research, New York.

Grossman, M. (1972b). "On the concept of health capital and the demand for health", Journal of Political Economy, Vol. 80; 223-255.

Heckman, J.J. (1979). "Sample selection bias as a specification error", Econometrica, Vol. 47; 153-161.

Idler, E.L. y Y. Benyamini (1997). "Self-Rated Health and Mortality: A Review of Twenty-Seven Community Studies", Journal of Health and Social Behavior, Vol. 38; 21-37.

Idler, E.L. y S. Kasl (1991). "Health perceptions and survival: Do global evaluations of health status really predict mortality?" Journal of Gerontology, Vol. 46; 55-65.

INDEC (2003). "La Salud a través de las Encuestas de Hogares en la Argentina", Serie Perfil de Condiciones de Vida No 2.

INE (1991-2000). Encuesta Continua de Hogares, Instituto Nacional de Estadística, Uruguay (http://www.ine.gub.uy).

Jewell, T., M. Rossi y P. Triunfo (2006). "El estado de salud del adulto mayor en América Latina", Documento de Trabajo 20/06, Departamento de Economía de la Facultad de Ciencias Sociales de la Universidad de la República.

Jewell, T., M. Rossi y P. Triunfo (2005). El estado de salud de los jóvenes uruguayos. Documento de Trabajo 13/05, Departamento de Economía de la Facultad de Ciencias Sociales de la Universidad de la República.

Long, J.S. y J. Freese (2001). Regression models for categorical dependent variables using STATA, Stata Press.

Mossey, J.M. y E. Shapiro (1982). "Self-Rated Health: a predictor of mortality among the elderly", American Journal of Public Health, Vol. 72; 800808.

Oswald, A.J. y C.M. Wilson (2005). "How Does Marriage Affect Physical and Psychological Health? A Survey of the Longitudinal Evidence", IZA Discussion Paper 1619, Institute for the Study of Labor.

Rhum, C.J. (2001). "Economic Expansions are Unhealthy: Evidence from Microdata", Working Paper 8447, National Bureau of Economic Research.

Rossi, M. y P. Triunfo (2004). "El Estado de Salud del Adulto Mayor en Uruguay", Documento de Trabajo 14/04, Departamento de Economía de la Facultad de Ciencias Sociales de la Universidad de la República.

Salas, C. (2002). "On the empirical association between poor health and low socioeconomic status at old age", Health Economics, Vol. 11; 207-220.

Statacorp. (2005). STATA Statistical Software: Release 9. College Station, TX: Statacorp LP.

Wallace, S. y V. Guitierrez (2005). "Equity of access to health care for older adults in four major Latin American cities", Pan American Journal of Public Health, Vol. 17; 394-409. 